\title{
3 Research Suare \\ Hidden Blood Loss and Factors in Shoulder Arthroscopy Surgery: A Review of 59 Patients
}

Nie Jiangbo

Zhejiang University Huzhou Hospital: Huzhou Central Hospital https://orcid.org/0000-0002-5639-8324

Mingchao Jin

Zhejiang University Huzhou Hospital: Huzhou Central Hospital

Tianshun Fang

Zhejiang University Huzhou Hospital: Huzhou Central Hospital

Xi Liu

Zhejiang University Huzhou Hospital: Huzhou Central Hospital

Haowei Jiang

Zhejiang University Huzhou Hospital: Huzhou Central Hospital

Jianyou Li ( $\sim$ ljywn1977@126.com )

Affiliated Huzhou Hospital, Zhejiang University School of Medicine, Huzhou 313000, Zhejiang province, China.

\section{Research article}

Keywords: Hidden blood loss, Shoulder arthroscopy, Risk factors, Multivariate linear regression analysis

Posted Date: March 24th, 2021

DOl: https://doi.org/10.21203/rs.3.rs-321579/v1

License: (c) (1) This work is licensed under a Creative Commons Attribution 4.0 International License.

Read Full License 


\section{Abstract}

Purpose: Subacromial impingement syndrome (SIS) and its related rotator cuff tears are the most important cause of shoulder joint pain, which has recently received greater attention. Arthroscopy is a

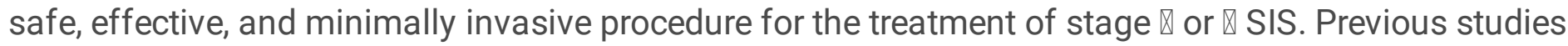
have reported that little blood loss usually occurs during this procedure. However, significant perioperative hidden blood loss (HBL) is often overlooked. In this respect, we herein aimed to investigate the amount of $\mathrm{HBL}$ and identify its possible risk factors.

Methods: We enrolled 59 patients with SIS who received shoulder arthroscopy between December 2019 and June 2020 in this study. The clinical data recorded included the height of patients, weight pre- and postoperative hematocrit (Hct), which were used to calculate HBL using Gross's formula. We analyzed the risk factors, including sex, age, BMI (body mass index), stage of SIS, diabetes, hypertension, and operative time using multivariate linear regression analysis.

Results: Our results revealed that the mean of HBL was $341.4 \pm 214.9 \mathrm{~mL}$, while that of the postoperative $\mathrm{Hb}$ loss was $13.3 \pm 8.0 \mathrm{~g} / \mathrm{L}$. The incidence of postoperative anemia was significantly associated with HBL $(X 2=14.496, P<0.001)$. Furthermore, multivariate linear regression analysis demonstrated that all seven factors, including gender $(P=0.698)$, age $(P=0.553)$, BMI $(P=0.854)$, stage of SIS $(P=0.906)$, diabetes $(P=0.984)$, hypertension $(P=0.532)$, and operative time $(P=0.645)$, were not significantly associated with $\mathrm{HBL}$.

Conclusion: Findings from this study show that postoperative HBL following shoulder arthroscopy was significant, which can aggravate anemia or lead to secondary anemia, thus should not be neglected.

\section{Introduction}

Subacromial impingement syndrome (SIS) and its related rotator cuff tears in adult patients are increasing steadily, severely affecting the quality of life of patients. In particular, they are the most significant causes of shoulder joint pain and are likely the outcomes of an age-related, degenerative process [1]. The primary goals of treatment are to relieve pain and restore function. According to Neer et al., SIS and its progression are characterized into three stages [2]. For early-stage impingement, conservative multimodal treatment is the most preferred option. On the other hand, for stage $\nabla$, stage $\nabla$ impingement, and rotator cuff tears, shoulder arthroscopic surgery (including arthroscopic acromioplasty or/and rotator cuff repair) is an effective and minimally invasive treatment $[3,4]$. Although little visible blood loss in minimally invasive surgeries is well documented, postoperative hemoglobin $(\mathrm{Hb})$ or hematocrit $(\mathrm{Hct})$ of patients undergoing those surgeries are found to be maintained at lower levels than the preoperative [5-7]. Thus, implying that lower postoperative $\mathrm{Hb}$ and $\mathrm{Hct}$ should be attributed to perioperative hidden blood loss (HBL), which is often overlooked in terms of research.

The concept of HBL was proposed by Sehat $e t$ al. [8] and has been widely studied. Previous investigations in the realm of orthopedics mostly focused on arthroplasty, posterior spine fusion surgery, 
and percutaneous kyphoplasty surgery (PKP), which showed that HBL was remarkable after surgery $[5,9$, 10]. Additionally, Wan et al. found that the mean of HBL after laparoscopic cholecystectomy (LC) was $259.3 \mathrm{ml}[6]$. These studies have enumerated that whatever in open or minimal surgery, HBL is a significant part of estimate total blood loss (TBL). Therefore, understanding HBL will help to estimate TBL more accurately during the perioperative period, which possibly can prevent related complications. Unfortunately, there is no published study considering HBL in shoulder arthroscopic surgery for rotator cuff disease. We thus speculated that shoulder arthroscopy can also lead to hidden blood loss. In this regard, we herein performed a retrospective review on patients who had received shoulder arthroscopy in our department to calculate the amount of $\mathrm{HBL}$, as well as to identify the influential factors causing $\mathrm{HBL}$ after shoulder arthroscopy surgery. Of note, in this study, we revealed that postoperative $\mathrm{Hb}$ in patients who received shoulder arthroscopy was lower in our clinical practice compared with the pre-operative.

\section{Materials And Methods}

\subsection{Patients}

All protocols and procedures used in this study were reviewed and approved by the Ethics Committee of Huzhou Hospital. The patients enrolled in this work provided informed consent. The inclusion criteria for enrollment were as follows: unilateral shoulder arthroscopy, degenerative injury, and complete medical data, while exclusion criteria included: traumatic rotator cuff injury, long-term bleeding disorders, blood transfusion throughout the assessment period, and multiple injuries. We retrospectively reviewed a total of 59 patients (23 males and 36 females) who had undergone shoulder arthroscopy between December 2019 and June 2020. Afterward, we recorded data which comprised of gender, age, weight, height, body mass index (BMI), the level of SIS, preoperative and postoperative hematocrit (Hct) and hemoglobin ( $\mathrm{Hb}$ ), operative time, hypertension, and diabetes mellitus.

\subsection{Surgical technique and postoperative therapy}

Based on the American Society of Anesthesiologists (ASA) physical score, all individuals received general anesthesia. The operation was performed with the patient lying in a lateral decubitus position by experienced surgeons (SZ). Hypotensive anesthesia, irrigated with epinephrine saline solution, and intraarticular tranexamic acid was applied to reduce bleeding and to provide a clear operative field. After the operation, the patients routinely received short-term postoperative analgesia.

\subsection{Management of blood loss}

Intraoperative visible blood loss was too little to be assessed. In addition, no patient underwent wound drainage. Therefore, we ignored it, and TBL was approximately considered as HBL. Notably, it is generally believed that fluid shifts have been largely completed and the hemodynamics of patients become stable on the second or third days after surgery [11]. Thus, a complete blood count including Hct and $\mathrm{Hb}$ of all patients were recorded before the operation and on the third postoperative day. 


\subsection{Calculation of the hidden blood loss}

We computed the patient's blood volume (PBV) as described by Nadler et al. as follows [12]:

PBV $(L)=k 1 \times$ height $(m) 3+k 2 \times$ weight $(k g)+k 3$,

where $\mathrm{k} 1=0.3669, \mathrm{k} 2=0.03219$, and $\mathrm{k} 3=0.6041$ are for males, whereas $\mathrm{k} 1=0.3561, \mathrm{k} 2=0.03308$, and $\mathrm{k} 3=0.1833$ are for females.

In this study, the TBL was estimated using the formula developed by Gross et al. as stated below [13]:

$\mathrm{TBL}(\mathrm{L})=\mathrm{PBV}(\mathrm{L}) \times\left(\left(\mathrm{Hct}_{\text {pre }}-\mathrm{Hct}_{\text {post }}\right)\right) / \mathrm{Hct}_{\text {ave }}$

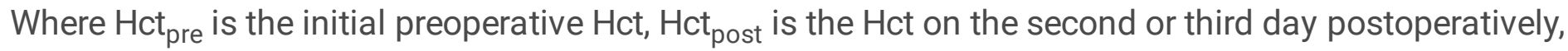
and $\mathrm{Hct}_{\text {ave }}$ is the average of $\mathrm{Hct}_{\text {pre }}$ and $\mathrm{Hct}_{\text {post }}$.

We recorded perioperative $\mathrm{Hb}$ so as to calculate Hbloss according to the following formula:

$\mathrm{Hb}_{\text {loss }}(\mathrm{g} / \mathrm{L})=\mathrm{Hb}_{\text {pre }}-\mathrm{Hb}_{\text {post }}$

\subsection{Level of SIS}

Here, we classified SIS into three stages as described by Neer et al.. In particular, patients with stage $\mathbb{Z}$ impingement underwent arthroscopic surgery after failure of conservative treatment. Subsequently, those patients with stage $\mathbb{Q}$ impingement were also included.

\subsection{Additional measurements}

A hemoglobin level of $<130 \mathrm{~g} / \mathrm{L}$ for men and $<120 \mathrm{~g} / \mathrm{L}$ for women was defined as anemia based on the World Health Organization (WHO) criteria [14].

\subsection{Statistical analysis}

All data analyses were implemented using SPSS software, version 26.0 (SPSS, Chicago, IL). A level of P< 0.05 was considered statistically significant. Mean \pm standard deviations were used as descriptive statistics. First, we used the Shapiro-Wilk test to assess the normality of variables, while the paired sample t-test was used to compare the differences between pre- and post-operative $\mathrm{Hb}$ levels and Hct values. We then examined the significant differences between the two stages using the independent sample' Student t-test and $\chi 2$ test. Additionally, we adopted the $\chi 2$ test to compare the pre- and postoperative incidence of anemia. Finally, multivariate linear regression analysis was performed to identify the influencing factors associated with $\mathrm{HBL}$, including three quantitative variables (age, BMI, and operative time) and four qualitative variables (sex, stage of SIS, diabetes, and hypertension). A positive coefficient indicates a positive influence on the dependent variable (HBL), whereas a negative coefficient reflects a negative influence. 


\section{Results}

Overall, 64 patients underwent shoulder arthroscopy between December 2019 and June 2020 in this work. Five patients were excluded: 1 patient with a traumatic injury, 1 patient (with shoulder joint infection), and 3 patients (with incomplete clinical data). Consequently, a total of 59 patients were enrolled in this study. As shown in Table 1, the average age was 60.6 years (range, 35 - 84 years), while that of BMI was 23.5 (range, $22.6-24.3$ ). In addition, the mean of postoperative HBL was $341.4 \pm 214.9$ $\mathrm{mL}$, whereas that of $\mathrm{Hb}$ loss was $13.3 \pm 8.0 \mathrm{~g} / \mathrm{L}$. The operative time of shoulder arthroscopy was $92.5 \pm$ $37.3 \mathrm{~min}$. Notably, a paired sample t-test revealed a significant difference between the values of pre- and post-operative $\mathrm{Hb}(\mathrm{P}<0.001)$.

There was no statistical difference in sex $(P=0.985)$, age $(P=0.118)$, height $(P=0.306)$, weight $(P=$ $0.223), \mathrm{BMI}(P=0.354)$, hypertension $(P=0.052)$ and diabetes $(0.363)$ between the two stages (Table 2$)$.

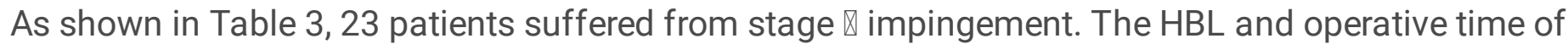
those patients were $347.5 \pm 192.1 \mathrm{~mL}$ and $71.1 \pm 35.2 \mathrm{~min}$, respectively. On the other hand, 12 patients

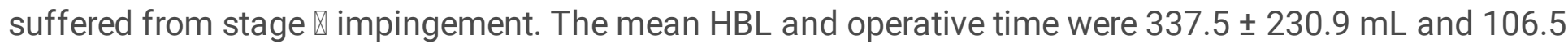
$\pm 31.8 \mathrm{~min}$, respectively. The increase in operation time was associated with the stage of SIS $(P<0.001)$. The procedure of rotator cuff repair in patients with stage $₫$ impingement prolonged the duration of

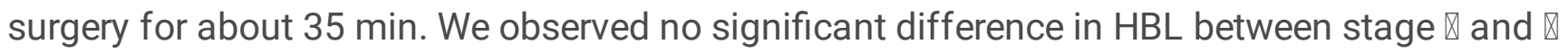
impingement $(P=0.863)$. Of note, 12 patients suffered from preoperative anemia, and subsequently, the number of patients with anemia after shoulder arthroscopy increased to 32 . We also recorded that $\mathrm{HBL}$ can significantly increase the risk of postoperative anemia $(X 2=14.496, P<0.001)$.

We further performed multiple linear regression analysis to examine the relationship between $\mathrm{HBL}$ and seven different influential factors. As is shown in Table 4, gender $(P=0.698)$, age $(P=0.553), B M I(P=$ $0.854)$, stage of SIS $(P=0.906)$, diabetes $(P=0.984)$, hypertension $(P=0.532)$, and operative time $(P=$ 0.645 ) were not significantly associated with HBL.

\section{Discussion}

As a new minimally invasive technique, arthroscopy exhibits the benefit of less visible blood loss during surgery. Unfortunately, HBL is often neglected by doctors in clinical practice. Previously, the perioperative bleeding volume has been demonstrated to be closely associated with postoperative complications, especially in old patients. Specifically, HBL occupies a significant proportion of perioperative bleeding, accounting for more than $50 \%$ of TBL [9]. Furthermore, excessive HBL increases early complications as well as affects functional reconstruction, which will lead to poor prognosis and dissatisfaction of patients $[15,16]$. Therefore, HBL in shoulder arthroscopy should be taken into consideration.

Herein, we found that the mean of HBL was $341.4 \pm 214.9 \mathrm{~mL}$, while that of $\mathrm{Hb}$ was $13.3 \pm 8.0 \mathrm{~g} / \mathrm{L}$ in the perioperative period. Besides, 20 patients whose preoperative $\mathrm{Hb}$ was normal developed secondary anemia. These results far exceeded the expected visible blood loss and were consistent with reports by 
Wu et al. and Wan et al [5, 6]. For patients with shoulder arthroscopy, initial injury and incomplete intraoperative hemostasis were the major causes of HBL. Elsewhere, it has been noted that anemia due to $\mathrm{HBL}$, particularly in the elderly, may increase postoperative mortality and morbidity.

To counteract these adverse effects, several previous studies focused on the mechanisms of HBL and found that $\mathrm{HBL}$ is possibly attributed to blood infiltration in tissue compartments and hemolysis.

However, the risk factors which may contribute to the rising levels of HBL remain unclear. In this study, we executed multiple linear regression analysis to detect the association factors. Surprisingly, we uncovered that all seven factors were unrelated to the amount of HBL. Previous studies have established that those factors were associated with a high level of HBL [5-11].

For as is well-known, more serious injuries will result in complex surgery and more blood loss. Herein, patients with stage $\otimes$ SIS suffered from degenerative rotator cuff tears. The procedure of rotator cuff repair prolonged the duration of surgery for approximately $35 \mathrm{~min}$. However, there was no statistical difference in HBL between the two stages. Additionally, serious injury and long operative time did not increase the HBL in shoulder arthroscopy. This result contradicted the findings reported by Cao et al. and Wan et al. [6, 17].

We further speculated whether several factors eliminated the influence of stage on HBL. To begin with, numerous reports have highlighted that the blood flow of cuffs with SIS has significantly decreased [18, 19]. Also, pathologies of SIS, such as sterile inflammation and calcification of the supraspinatus tendon, may impair cuffs tissue and affect its blood supply. Elsewhere, another study elucidated that cuff groups

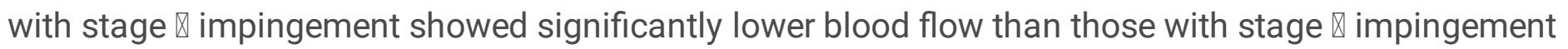
[19]. Besides, suture anchors were applied for repairing the rotator cuff tears in patients with stage $\nabla$ SIS, which resulted in a significant decline in the blood flow of cuffs at the initial time point of fixation [20].

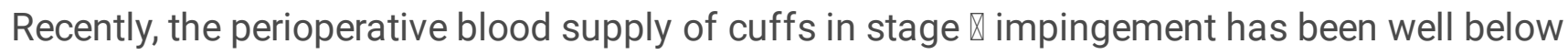
expectations. Notably, irrigation with epinephrine saline and application of tranexamic acid significantly reduced postoperative intra-articular bleeding $[20,21]$.

Furthermore, in the present study, we found no association between hypertension and $\mathrm{HBL}$, and this finding was parallel to the results by Wu et al. [5]. However, this conclusion is still under discussion. A study by Wan et al. demonstrated that hypertension significantly increased HBL [6]. Whether the operative region involves major arteries may disturb the influence that hypertension on the HBL. For example, the procedure of LC involving ligation of the cystic artery, vascular fragility caused by long-term hypertension may increase postoperative bleeding [6]. However, other operations such as PKP and cervical open-door laminoplasty primarily affect blood capillaries and tiny vessels, which may explain why postoperative HBL was no associated with hypertension $[7,17]$.

Moreover, we herein revealed that $\mathrm{HBL}$ was unaffected by gender, age, BMI, and diabetes mellitus. This finding was congruent with studies on LC and PKP, likely because of similarity in the concept of minimally invasive surgery techniques $[5,6]$. 
However, despite these intriguing results, this study exhibits several shortcomings. First, the number of patients included in this retrospective study was insufficient, and the operations were not performed by the same surgeon. Thus, we recommend to future prospective studies to incorporate an adequate number of patients. Second, we used Hct on the third day after surgery to evaluate HBL, because previous research suggested that the fluid shifts of patients would be complete at this time. However, we cannot guarantee that this way was optimum. Finally, visible bleeding was overlooked in this study. The amount of HBL that we calculated was inaccurate, which may have affected our investigation.

In conclusion, this study demonstrates that postoperative HBL following shoulder arthroscopy was significant, which can aggravate anemia or lead to secondary anemia, thus should not be overlooked.

\section{Abbreviations}

HBL: Hidden blood loss; TBL: Total blood loss; SIS: Subacromial impingement syndrome; Hct: hematocrit; $\mathrm{Hb}$ : hemoglobin; BMI: Body mass index; PKP: percutaneous kyphoplasty surgery; LC: laparoscopic cholecystectomy; SZ: experienced surgeons.

\section{Declarations}

\section{Acknowledgement}

Thanks to my senior, Yongli Wang, for his contribution in the data analysis.

\section{Authors' contributions}

$\mathrm{JN}, \mathrm{MJ}, \mathrm{TF}, \mathrm{XL}, \mathrm{HJ}$, and $\mathrm{JL}$ were all with the conception and design of the study or acquisition of data, or analysis, and interpretation of data, and drafting the article and revising it. All authors read and approved the final manuscript.

\section{Funding}

Not applicable

\section{Availability of data and materials}

The datasets used and/or analyzed during the current study are available from the corresponding author on reasonable request.

\section{Ethics approval and consent to participate}

All protocols and procedures used in this study were reviewed and approved by the Ethics Committee of Huzhou Central Hospital. 
All authors have seen the manuscript and approved to submit to your journal.

\section{Competing interests}

On behalf of all authors, the corresponding author states that there is no conflict of interest.

\section{References}

1. Page MJ, Huang H, Verhagen AP, Gagnier JJ, Buchbinder R. Outcome Reporting in Randomized Trials for Shoulder Disorders: Literature Review to Inform the Development of a Core Outcome Set. Arthritis Care Res (Hoboken). 2018;70(2):252-259. doi:10.1002/acr.23254.

2. Neer CS 2nd. Anterior acromioplasty for the chronic impingement syndrome in the shoulder. 1972. J Bone Joint Surg Am. 2005;87(6):1399. doi:10.2106/JBJS.8706.cl.

3. Karjalainen TV, Jain NB, Heikkinen J, Johnston RV, Page CM, Buchbinder R. Surgery for rotator cuff tears. Cochrane Database Syst Rev. 2019;12(12):CD013502. Published 2019 Dec 9. doi:10.1002/14651858.CD013502.

4. Paavola M, Malmivaara A, Taimela S, et al. Subacromial decompression versus diagnostic arthroscopy for shoulder impingement: randomised, placebo surgery controlled clinical trial. BMJ. 2018;362:k2860. Published 2018 Jul 19. doi:10.1136/bmj.k2860.

5. Wu YS, Zhang H, Zheng WH, Feng ZH, Chen ZX, Lin Y. Hidden blood loss and the influential factors after percutaneous kyphoplasty surgery. Eur Spine J. 2017;26(7):1878-1883. doi:10.1007/s00586017-4950-9.

6. Wan RR, Wang YL, Wu XC, et al. Hidden blood loss and the influencing factors after laparoscopic cholecystectomy. ANZ J Surg. 2020;90(1-2):103-108. doi:10.1111/ans.15502.

7. Jiang $C$, Chen TH, Chen ZX, Sun ZM, Zhang H, Wu YS. Hidden blood loss and its possible risk factors in cervical open-door laminoplasty. J Int Med Res. 2019;47(8):3656-3662. doi:10.1177/0300060519856987.

8. Sehat KR, Evans R, Newman JH. How much blood is really lost in total knee arthroplasty?. Correct blood loss management should take hidden loss into account. Knee. 2000;7(3):151-155. doi:10.1016/s0968-0160(00)00047-8.

9. Guo WJ, Wang JQ, Zhang WJ, Wang WK, Xu D, Luo P. Hidden blood loss and its risk factors after hip hemiarthroplasty for displaced femoral neck fractures: a cross-sectional study. Clin Interv Aging. 2018;13:1639-1645. Published 2018 Sep 10. doi:10.2147/CIA.S174196.

10. Hu Y, Li Q, Wei BG, et al. Blood loss of total knee arthroplasty in osteoarthritis: an analysis of influential factors. J Orthop Surg Res. 2018;13(1):325. Published 2018 Dec 22. doi:10.1186/s13018018-1038-0.

11. Sehat KR, Evans RL, Newman JH. Hidden blood loss following hip and knee arthroplasty. Correct management of blood loss should take hidden loss into account. J Bone Joint Surg Br. 2004;86(4):561-565. 
12. Nadler SB, Hidalgo JH, Bloch T. Prediction of blood volume in normal human adults. Surgery. 1962;51(2):224-232.

13. Gross JB. Estimating allowable blood loss: corrected for dilution. Anesthesiology. 1983;58(3):277280. doi:10.1097/00000542-198303000-00016.

14. Beghé $C$, Wilson A, Ershler WB. Prevalence and outcomes of anemia in geriatrics: a systematic review of the literature. Am J Med. 2004;116 Suppl 7A:3S-10S. doi:10.1016/j.amjmed.2003.12.009.

15. Smorgick Y, Baker KC, Bachison CC, Herkowitz HN, Montgomery DM, Fischgrund JS. Hidden blood loss during posterior spine fusion surgery. Spine J. 2013;13(8):877-881. doi:10.1016/j.spinee.2013.02.008.

16. Liu X, Zhang X, Chen Y, Wang Q, Jiang Y, Zeng B. Hidden blood loss after total hip arthroplasty. J Arthroplasty. 2011;26(7):1100-5.e1. doi:10.1016/j.arth.2010.11.013.

17. Cao D, Zhang S, Yang F, Shen K, Tan Z. Hidden blood loss and its influencing factors after percutaneous kyphoplasty surgery: A retrospective study. Medicine (Baltimore). 2018;97(15):e0435. doi:10.1097/MD.0000000000010435.

18. Levy O, Relwani J, Zaman T, Even T, Venkateswaran B, Copeland S. Measurement of blood flow in the rotator cuff using laser Doppler flowmetry. J Bone Joint Surg Br. 2008;90(7):893-898. doi:10.1302/0301-620X.90B7.19918.

19. Karthikeyan S, Griffin DR, Parsons N, et al. Microvascular blood flow in normal and pathologic rotator cuffs. J Shoulder Elbow Surg. 2015;24(12):1954-1960. doi:10.1016/j.jse.2015.07.014.

20. Christoforetti JJ, Krupp RJ, Singleton SB, Kissenberth MJ, Cook C, Hawkins RJ. Arthroscopic suture bridge transosseus equivalent fixation of rotator cuff tendon preserves intratendinous blood flow at the time of initial fixation. J Shoulder Elbow Surg. 2012;21(4):523-530.

21. Tsukada S, Kurosaka K, Nishino M, Maeda T, Hirasawa N, Matsue Y. Intraoperative Intravenous and Intra-Articular Plus Postoperative Intravenous Tranexamic Acid in Total Knee Arthroplasty: A PlaceboControlled Randomized Controlled Trial. J Bone Joint Surg Am. 2020;102(8):687-692. doi:10.2106/JBJS.19.01083.

\section{Tables}

Table 1 Patients' demographic information and clinical results in patients with shoulder arthroscopy 


\begin{tabular}{|ll|}
\hline Paraments & Mean \pm SD \\
\hline Number of patients & 59 \\
\hline Age, years & $60.6 \pm 9.5$ \\
\hline Height, $\mathrm{m}$ & $1.61 \pm 0.09$ \\
\hline Weight, $\mathrm{kg}$ & $61.7 \pm 12.2$ \\
\hline BMI, kg/m² & $23.5 \pm 3.3$ \\
\hline Hemoglobin loss, g/L & $13.3 \pm 8.0$ \\
\hline Hematocrit level loss, \% & $9.0 \pm 5.7$ \\
\hline Hemoglobin level loss, \% & $10.3 \pm 6.2$ \\
\hline Operative time, min & $92.5 \pm 37.3$ \\
\hline Hidden blood loss, $\mathrm{mL}$ & $341.4 \pm 214.9$ \\
\hline
\end{tabular}

Data are mean $\pm S D$

$\mathrm{BMI}$ are body mass index

Table 2 Patients' demographic information of two stages

\begin{tabular}{|c|c|c|c|}
\hline Parameters & Stage $\otimes(n=23)$ & 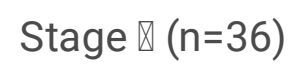 & $\mathrm{p}$ \\
\hline Sex, n & & & 0.985 \\
\hline Male & 9 & 14 & \\
\hline Female & 14 & 22 & \\
\hline Age, years & $58.2 \pm 10.0$ & $62.2 \pm 8.9$ & 0.118 \\
\hline Height, m & $1.63 \pm 0.07$ & $1.61 \pm 0.09$ & 0.306 \\
\hline Weight, kg & $64.1 \pm 11.9$ & $60.1 \pm 12.3$ & 0.223 \\
\hline BMI & $24.0 \pm 3.2$ & $23.1 \pm 3.4$ & 0.354 \\
\hline Hypertension, $\mathrm{n}$ & 3 & 13 & 0.052 \\
\hline Diabetes, n & 1 & 4 & 0.363 \\
\hline
\end{tabular}

Data are mean $\pm S D$

BMl are body mass index 
Table 3 Number and involved levels

\begin{tabular}{|llll|}
\hline Neer grade & Number of patients & Hidden blood loss, $\mathrm{mL}$ & Operation time, min \\
\hline Stage II & 23 & $347.5 \pm 192.1$ & $71.1 \pm 35.2$ \\
\hline Stage III & 36 & $337.5 \pm 230.9$ & $106.5 \pm 31.8$ \\
\hline P & & 0.863 & 0.000 \\
\hline
\end{tabular}

Data are mean $\pm S D$

Table 4 Results of multiple linear regression analysis for $\mathrm{HBL}$ coefficients

\begin{tabular}{|lllllll|}
\hline & \multicolumn{2}{ll|}{ Unstandardized coefficients } & Standardized coefficients & & \\
\hline (constant) & $\beta$ & SE & $\beta$ & $T$ & $P$ \\
\hline Sex & 148.32 & 380.54 & & 0.390 & 0.698 \\
\hline Age, years & 76.10 & 62.82 & 0.174 & 1.211 & 0.231 \\
\hline BMI, Kg/m & 2.22 & 3.71 & 0.097 & 0.597 & 0.553 \\
\hline Stage of SIS & -0.57 & 9.78 & -0.009 & -0.058 & 0.954 \\
\hline Diabetes & 8.51 & 72.01 & 0.019 & 0.118 & 0.906 \\
\hline Hypertension & 2.12 & 107.47 & 0.003 & 0.020 & 0.984 \\
\hline Operative time, $\min$ & -0.44 & 0.94 & -0.075 & -0.629 & 0.532 \\
\hline
\end{tabular}

Dependent variable: $\mathrm{HBL}(\mathrm{mL})$

BMl: body mass index 